\title{
Flat Reflectarray that Generates Adjacent Beams by Discriminating in Dual Circular Polarization
}

Rafael Florencio, José A. Encinar,

Giovanni Toso
Rafael R. Boix,
Mariano Barba, and

\begin{abstract}
One planar $18 \times 18 \mathrm{~cm}^{2}$ reflectarray prototype, capable of generating adjacent pencil beams with orthogonal circular polarizations (CP) in Ka-band (19.2-20.2GHz), has been designed, manufactured and measured as a proof-of-concept to demonstrate the possibility of beam separation in dual CP applications. The prototype exhibits main beams pointing at elevation angles of $16.7^{\circ}$ and $21.3^{\circ}$ for left-hand CP and righthand CP respectively. A $1.5 \mathrm{~dB}$ gain variation and $66 \%$ of efficiency is achieved in the frequency band 19.2-20.2GHz for a design center frequency of $19.7 \mathrm{GHz}$. These results will be useful for future work involving the design of multi-spot dual CP and dual band planar reflectarrays.
\end{abstract}

Index Terms - Satellite communications, Dipole arrays, circular polarization, dual-polarized antennas, reflectarrays.

\section{INTRODUCTION}

$\mathrm{O}_{\mathrm{for}}$ VER the last few decades, multiple-beam antenna systems for satellite communications have been very demanded. Multiple-beam antennas are currently being used for direct-broadcast satellites, personal communication satellites, military communication satellites, and high-speed Internet applications. These antennas provide mostly contiguous coverage over a specified field of view on Earth by using high-gain multiple spot beams for transmit and receive coverage [1].

In order to avoid mutual interference between the spot beams, a four-color frequency and polarization reuse scheme is usually employed [1]-[2]. In this scheme two different frequency sub-bands and two orthogonal polarizations are used in such a way that each spot in two adjacent colors could differ in frequency, polarization or both [3]. Therefore, the spots can transfer different information without mutual interferences. Spots with the same color use the same frequency and the same polarization, but they are spatially isolated from each other, so no spot has a neighbor with the same color

In recent years, a significant effort has been carried out in reducing the number of main apertures which generate the multiple spot beam coverage. Several solutions involving reflector antennas have been addressed [3]. Planar printed reflectarray antennas [4]-[5] have proven to be a potential alternative to shaped curved reflectors antennas thanks to low-profile, low mass and reduced manufacturing time and cost. Dual linear polarization printed planar reflectarrays have been designed which make it possible to achieve any value of phase-shift independently for each polarization, which can be used to generate different beams in each polarization within the same frequency band [6]-[8]. Also, printed planar reflectarrays offer the capability to generate pencil beams with the same beam direction which operate in two frequency bands for single polarization in each frequency band [9]-[13]. In particular, in [9] and [10] different single-layer multiresonant elements are used to generate a focused beam for left-hand circular polarization (LHCP) at $20 \mathrm{GHz}$ and a focused beam for right-hand circular polarization (RHCP) at $30 \mathrm{GHz}$. In [11] an element based on rectangular stacked patches is used to design a dual band pencil beam reflectarray operating at $6.5 \mathrm{GHz}$ and $10.5 \mathrm{GHz}$ with the same linear polarization. In [12] a dual-band $\mathrm{X} / \mathrm{Ka}$ reflectarray with single linear polarization has been implemented by placing a Kaband reflectarray backed by an FSS on top of an X-band reflectarray. This strategy has been extended in [13] for the design of a circularly polarized tri-band reflectarray which operates at $1.6 \mathrm{GHz}, 20.0 \mathrm{GHz}$ and $29.8 \mathrm{GHz}$ with RHCP, LHCP and RHCP respectively. However, despite the results of [12] and [13] are satisfactory, the antennas proposed in these two works require thick layers and three metallization levels, which make them bulky and difficult to manufacture.

Recent works [14]-[15] have shown the capabilities of planar reflectarrays made of sets of orthogonal dipoles to generate independent beams in orthogonal linear polarizations in two frequency bands (for $\mathrm{K} / \mathrm{Ka}$ frequency bands in [14], and $\mathrm{Ku} / \mathrm{K}$ bands in [15]). Unfortunately, the generation of 
independent beams in dual circular polarization (CP) is not an easy task. A reflectarray cell was proposed in [16] to provide independent phasing in each CP. The cells were made of two printed layers separated $20-\mathrm{mm}$ and enclosed in metallic cavities to avoid coupling. Whereas the upper layer is a $\mathrm{CP}$ selective surface with vertical bias that reflects LHCP waves and transmit RHCP waves with a 2-bit phase resolution, the lower layer is a star-like dipole configuration that reflects RHCP waves, also with a 2-bit phase resolution. A small size reflectarray made of 97 cells arranged in a 11 by 11 grid was used to demonstrate the generation of independent beams in dual-CP with $9.4 \%$ bandwidth at $8.37 \mathrm{GHz}$ [17]. Although the results were satisfactory, the geometry is bulky and the manufacturing process is complicated because of the presence of metallic cavities to isolate the cells, and because of the existence of different layers and spacers, one of the layers including vertical bias. Therefore, this configuration does not seem to be very suitable for multi-beam antennas used in space applications.

In [3] a parabolic reflectarray was designed to generate two adjacent beams in dual-CP at $20 \mathrm{GHz}$. In this design, while the parabolic surface was used to collimate the beam, the introduction of the reflectarray with variable rotation angles of the elements was used to separate the pointing directions of the pencil beams for LHCP and RHCP. In this work we demonstrate that it is possible to obtain separate beams in dual $\mathrm{CP}$ by using a flat reflectarray to both collimate the beam and discriminate in CP. This result will be useful for future work aimed at designing multi-spot dual $\mathrm{CP}$ and dual band planar reflectarrays.

As a proof-of-concept, in order to separate the LHCP and RHCP beams generated by a planar reflectarray, in this work we will use the element based on two orthogonal sets of three parallel dipoles described in [8]. It has been already shown that this element makes it possible an independent control of the beams in dual linear polarization applications [8], while requiring a simple manufacturing process. The elements will be rotated to generate two different pencil beams in dual CP at $19.7 \mathrm{GHz}$. Section II will show how the rotation angles of the elements can be used to separate the reflection phases in LHCP and RHCP. In section III, a conventional adjustment of the dipoles lengths will be carried out in a preliminary design to radiate pencil beams in the same direction for dual circular polarizations. Then, the variable rotation angles of the elements of the reflectarray will introduce the required phaseshift in circular polarization to split the beam directions by discriminating in circular polarization. Finally, experimental results and conclusions will be provided in sections IV and $\mathrm{V}$ respectively.

\section{REFLECTARRAY ELEMENT}

The reflectarray proposed in this paper is made of reflectarray elements based on two orthogonal sets of three parallel dipoles [8] as shown in Fig. 1(a). When the dipoles are parallel to the boundaries of the unit cell as in Fig. 1(a), one set of dipoles is introduced to control the phase-shift in one linear polarization, and the other set is introduced to control the phase-shift in the orthogonal linear polarization.

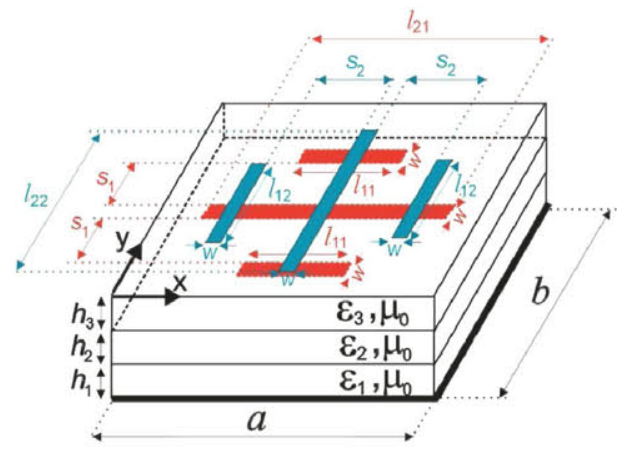

(a)

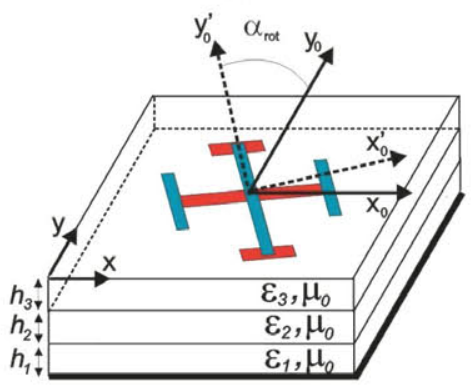

(b)

Fig. 1. (a) Reflectarray element based on two orthogonal sets of three coplanar parallel dipoles without rotation. (b) Reflectarray element with rotated dipoles and definition of rotation angle $\alpha_{\text {rot }}$.

Both set of dipoles are printed on the opposite sides of the upper dielectric layer. A bottom dielectric layer is used as separator with the ground plane, and a third bonding layer is used between the upper layer and the separator. The material used for the upper layer and the separator is a commercial low loss material to reduce the RF losses, $\operatorname{Diclad}^{\mathrm{TM}} 880$. Honeycomb can also be used instead as a separator to reduce the mass in space antennas. Thermoplastic bonding film 6250 is used as bonding layer. So, the multilayered substrate supporting the reflectarray element is composed of three dielectric layers with thickness $h_{i}$ and complex permittivity $\varepsilon_{i}=\varepsilon_{0} \varepsilon_{r i}\left(1-j \cdot \tan \delta_{i}\right)-i=1,2,3$ - (see Fig. 1(a)), where $\varepsilon_{r 1}=2.3, \varepsilon_{r 2}=$ 2.32, $\varepsilon_{r 3}=2.17, \tan \delta_{1}=0.0025, \tan \delta_{2}=0.0013, \tan \delta_{3}=0.002$, $h_{1}=1.524 \mathrm{~mm}, h_{2}=0.076 \mathrm{~mm}, h_{3}=0.127 \mathrm{~mm}$. A period of $a=b=7.2 \mathrm{~mm}$ (which is roughly $0.47 \lambda$ at $19.7 \mathrm{GHz}$ ) has been selected to provide both enough room for the dipoles and enough range of phase-shift at the design frequency and, at the same time, to avoid the appearance of grating lobes. The rest of the geometrical parameters have been adjusted to provide a smooth and linear behavior of the phase-shift for variations of the lengths of the dipoles, the final dimensions being $l_{11}=$ $0.7 l_{21}, l_{12}=0.7 l_{22}, w=0.5 \mathrm{~mm}, s_{1}=s_{2}=1.9 \mathrm{~mm}$.

In order to provide an additional phase-shift in $\mathrm{CP}$, in this paper the dipoles of Fig. 1(a) will be rotated as shown in Fig. 1(b). A home-made electromagnetic code that applies the Method of Moments in the Spectral Domain (MoM-SD) is used for the analysis of the reflectarray cell with rotated dipoles of Fig. 1(b) in a periodic environment. Basis functions accounting for edge singularities are used in the approximation of the current density of the dipoles [18]. The Fourier Transforms of these basis functions on the rotated 
dipoles are computed as described in [19]. The reflectarray cell is characterized by means of the reflection matrix $\mathbf{R}$, which includes the co-polar and cross-polar reflection coefficients for the two linear polarizations which are parallel to the axes $\mathrm{x}_{0}$ and $\mathrm{y}_{0}$ shown in Fig. 1(b). The $\mathbf{R}$ matrix relating the cartesian complex components of the reflected and incident tangential electric field on the reflectarray cell in a periodic environment can be written as shown below:

$$
\left(\begin{array}{l}
E_{x}^{r e f} \\
E_{y}^{r e f}
\end{array}\right)=\left(\begin{array}{ll}
R_{x x} & R_{x y} \\
R_{y x} & R_{y y}
\end{array}\right) \cdot\left(\begin{array}{l}
E_{x}^{i n c} \\
E_{y}^{i n c}
\end{array}\right)
$$

The complex z-component of the reflected electric field is computed by the known plane wave relation as shown below:

$$
E_{z}^{\text {rof }}=-\frac{k_{0} \sin \theta_{i} \cos \phi_{i} E_{x}^{\text {rof }}+k_{0} \sin \theta_{i} \sin \phi_{i} E_{y}^{\text {rof }}}{k_{0} \cos \theta_{i}}
$$

where $k_{0}$ is the propagation constant in vacuum, and $\theta_{i}$ and $\phi_{i}$ are the spherical angular coordinates of the incident direction of the plane wave impinging on the periodic cell.

In the same way, a matrix $\mathbf{R}^{\prime}$ can be defined relating the cartesian complex components of the reflected and incident tangential electric with respect to the axes $x^{\prime}{ }_{0}$ and y' ${ }_{0}$ of Fig. 1(b). Bearing in mind that the axes $x^{\prime}{ }_{0}$ and $y^{\prime}{ }_{0}$ are the result of rotating the axes $\mathrm{x}_{0}$ and $\mathrm{y}_{0}$ an angle $\alpha_{\text {rot }}$ around the $\mathrm{z}$ axis, $\mathbf{R}^{\prime}$ can be obtained in terms of $\mathbf{R}$ as shown below:

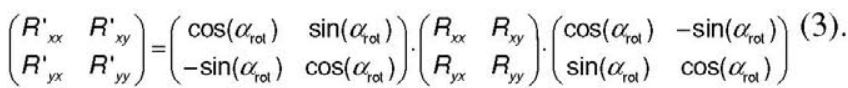

The right and left CP complex components of the reflected and incident electric fields $-\mathrm{E}_{\mathrm{RHCP}}=\mathrm{E}_{\theta^{+}}+\mathrm{j} \cdot \mathrm{E}_{\phi}$ and $E_{\mathrm{LHCP}}=\mathrm{E}_{\theta}-\mathrm{j} \cdot \mathrm{E}_{\phi^{-}}$can be obtained in terms of the complex components in spherical coordinates $\mathrm{E}_{\theta}$ and $\mathrm{E}_{\phi}$. And $\mathrm{E}_{\theta}$ and $\mathrm{E}_{\phi}$ can be obtained in terms of the cartesian components $E_{x}, E_{y}$, and $E_{2}$ by means of the transformations shown in [20]. So, we can define a matrix $\mathbf{R}^{\mathbf{c}}$ which relates the complex $\mathrm{CP}$ components of the reflected and incident tangential electric field on the reflectarray cell in a periodic environment as shown below:

$$
\left(\begin{array}{l}
E_{R H C P}^{r e f} \\
E_{L H C P}^{r e f}
\end{array}\right)=\left(\begin{array}{ll}
R_{R H C P, R H C P} & R_{R H C P, L H C P} \\
R_{L H C P, R H C P} & R_{L H C P, L H C P}
\end{array}\right) \cdot\left(\begin{array}{l}
E_{R H C P}^{\text {inc }} \\
E_{L H C P}^{\text {inc }}
\end{array}\right)
$$

The reflection coefficients $\mathrm{R}_{\mathrm{xx}}$ and $\mathrm{R}_{\mathrm{yy}}$ have been computed at different frequencies as a function of the dipole lengths when $l_{22}=l_{21}=l$ in the case where the dipoles are not rotated $\left(\alpha_{\text {rot }}=0^{\circ}\right)$. Fig. 2 shows the results for the magnitude and phase of $\mathrm{R}_{\mathrm{xx}}$ under oblique incidence $\left(\theta_{i}=19^{\circ}, \phi_{i}=0^{\circ}\right)$. Similar curves have been obtained for $\mathrm{R}_{\mathrm{yy}}$. The results show low level of losses (lower than $0.2 \mathrm{~dB}$ ) and very linear and smooth behavior of the phase curves with phase range larger than $400^{\circ}$. If a reflectarray antenna is to be designed, the phase curves for $R_{x x}$ and $R_{y y}$ can be used to adjust the dipole dimensions in each reflectarray cell that introduce the required phase-shift for the generation of a pencil beam in dual linear polarization when the reflectarray is fed by a linearly polarized feeder [4], [8].
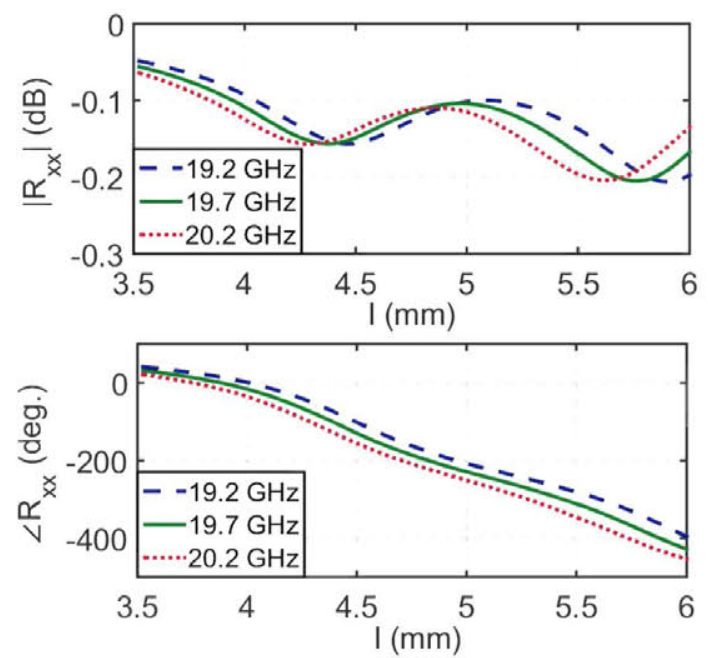

Fig. 2. Magnitude and phase of the cell reflection coefficient $R_{x x}$ with respect to the length of dipoles at different frequencies under oblique incidence $\left(\theta_{\mathrm{i}}=\right.$ $19^{\circ}, \phi_{\mathrm{i}}=0^{\circ}$ ) when $\alpha_{\text {rot }}=0^{\circ}$.

In Ref. [21], Huang and Pogorzelski qualitatively demonstrated that the phases of the coefficients $R_{\text {RHCP,RHCP }}$ and $\mathrm{R}_{\mathrm{LHCP}, \mathrm{LHCP}}$ defined in (4) have a linear dependence on the rotation angle $\alpha_{\text {rot }}$ defined in Fig. 1(b). This linear dependence can be written as

$$
\angle R_{\text {RHCP, RHCP }}=\psi_{0}+2 \alpha_{\text {rot }}, \angle R_{\text {LHCP,LHCP }}=\psi_{0}-2 \alpha_{\text {rot }}
$$

where $\psi_{0}$ is the phase of $\mathrm{R}_{\mathrm{RHCP}, \mathrm{RHCP}}$ and $\mathrm{R}_{\mathrm{LHCP}, \mathrm{LHCP}}$ when the reflectarray element isn't rotated. The demonstration of (5) given in [21] was not rigorous, and was only provided for a wave normally incident on the periodic structure containing the reflectarray element. Based on this demonstration, a planar reflectarray made of identical patches having variable rotation angles was designed in [21] to generate a circularly polarized pencil beam, and the concept worked pretty well. The concept of element rotation for CP generation was revisited in [3], where a parabolic reflectarray made of dipoles was designed to generate two independent beams with orthogonal CPs. In this latter case, the element rotation concept was used to separate the two circularly polarized beams. In principle, the rotations of the elements were carried out in accordance with (5). However, the authors of [3] comment that the lengths of the dipoles had to be adjusted after angle rotation in each reflectarray cell in order to compensate for the effect of the different incidence angles.

Fig. 3 (a) shows the difference between the phases of $R_{x x}^{\prime}$ and $\mathrm{R}_{\text {yy }}$ when the dipoles of Fig. 1(b) are rotated as a function of the rotation angle $\alpha_{\text {rot }}$. The lengths of the dipoles of each orthogonal set have been adjusted to provide $180^{\circ}$ of difference of phase $\left(l_{21}=5.8 \mathrm{~mm} ; l_{22}=4.98 \mathrm{~mm}\right)$ when the dipoles aren't rotated. Note that this phase difference in the absence of rotation was explicitly enforced in Eqns. (3) and (5) of [21] before the elements were rotated for the generation of CP. The results of Fig. 3 (a) show that the phase difference 
departs from $180^{\circ}$ when the dipoles rotate, reaching a maximum departure of $35^{\circ}$ with respect to the $180^{\circ}$ value.

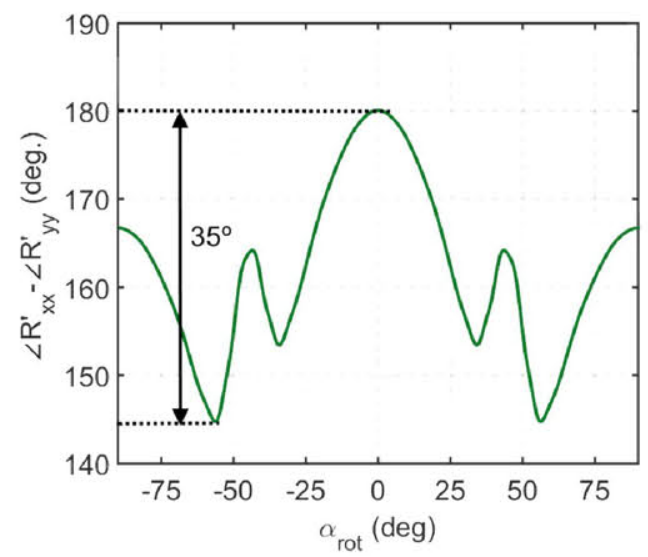

(a)

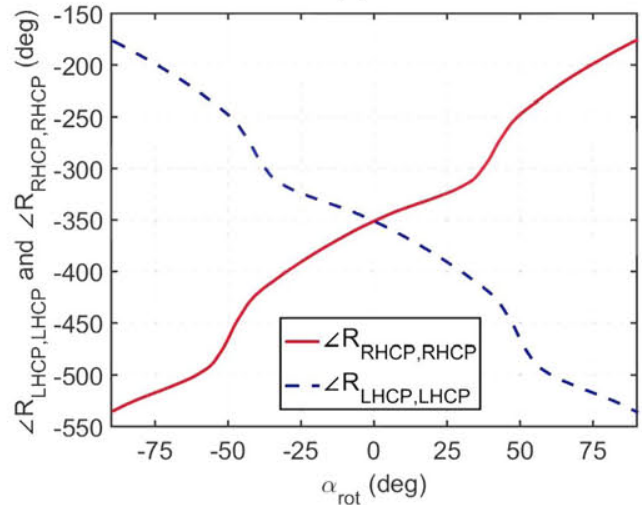

(b)

Fig. 3. (a) Difference of phase between $\mathrm{R}^{\prime}{ }_{x \mathrm{x}}$ and $\mathrm{R}_{\mathrm{yy}}$ as a function of the rotation angle. (b) Phase of $R_{R H C P, R H C P}$ and $R_{L H C P, L H C P}$ as a function of the rotation angle. Both analysis are made with $l_{21}=5.8 \mathrm{~mm}$ and $l_{22}=4.98 \mathrm{~mm}$ under oblique incidence $\left(\theta_{\mathrm{i}}=19^{\circ} \phi_{\mathrm{i}}=0^{\circ}\right)$.

Fig. 3 (b) shows the phases of $\mathrm{R}_{\mathrm{RHCP}, \mathrm{RHCP}}$ and $\mathrm{R}_{\mathrm{LHCP}, \mathrm{LHCP}}$ as a function of the rotation angle $\alpha_{\text {rot }}$. These results show a roughly linear behavior of the phase curves for small rotations. However, the behavior of the phases of $\mathrm{R}_{\mathrm{RHCP}, \mathrm{RHCP}}$ and $\mathrm{R}_{\mathrm{LHCP}, \mathrm{LHCP}}$ departs from linearity as $\left|\alpha_{\text {rot }}\right|$ increases, which is due to the fact that the difference between the phases of $\mathrm{R}_{\mathrm{xx}}$ and $R_{\text {yy }}^{\prime}$ departs from $180^{\circ}$ in Fig. 3 (a) up to a maximum value of $35^{\circ}$. Although a linear behavior would be desirable for the design of a circularly polarized reflectarray based on element rotation [21], the results of Fig. 3(b) indicate that Eqn. (5) is just a very coarse approximation. The shape of the curves shown in Fig. 3(b) change as the angles of incidence and the dimensions of the dipoles change, which is something to be taken into account in a reflecterray design where the angles of incidence and the dimensions of the dipoles change from cell to cell. One interesting thing about Fig. 3(b) is that despite the non-linearity of the curves, a rotation angle $\alpha_{\text {rot }}$ of the reflectarray element will introduce opposite sign shifts of roughly the same magnitude in the phases of the coefficients $\mathrm{R}_{\mathrm{RHCP}, \mathrm{RHCP}}$ and $\mathrm{R}_{\mathrm{LHCP}, \mathrm{LHCP}}$ with respect to the non-rotated case. In the next section this idea will be exploited in a planar reflectarray design to generate two independent pencil beams with orthogonal circular polarization. First, an adjustment of the dipoles lengths will be carried out in a preliminary design to generate radiated pencil beams in the same direction for dual linear polarizations. This means that in case the feeder is circularly polarized, the designed reflectarray will generate pencil beams with the same beam direction for dual circular polarization. Once this pre-design is finished, we will compute the values of the phases of $R_{R H C P, R H C P}$ and $R_{L H C P, L H C P}$ that are required to separate the directions of the dual circular polarization beams by means of conventional reflectarray theory [4]. Then, the required values of $\alpha_{\text {rot }}$ for each element will be obtained in terms of the necessary shifts for the phases of $\mathrm{R}_{\mathrm{RHCP}, \mathrm{RHCP}}$ and $\mathrm{R}_{\mathrm{LHCP}, \mathrm{LHCP}}$. In this latter step, we will not use the approximation (5) because it is not accurate enough. Instead, real phase shift curves of the type shown in Fig. 3(b) will be used for each reflectarray element.

\section{REFLECTARRAY DESIGN TO GENERATE TWO INDEPENDENT RADIATED BEAMS IN DUAL CIRCULAR POLARIZATION}

In this section, we present the design of a planar reflectarray capable to radiate two collimated beams at dual CPs at a center frequency of $19.7 \mathrm{GHz}$. The reflectarray consists of $25 \times 25$ elements, and it is based on the element with two orthogonal sets of rotated dipoles (see Fig. 1(b)) described in Section II.

\section{A. Experimental characterization of the corrugated feed- horn}

In order to feed the designed reflectarray, we use a circularly polarized corrugated feed horn operating in the frequency band 19.2-20.2GHz. The phase centre of the horn is located at the coordinates $\left(\mathrm{x}_{\mathrm{f}}, \mathrm{y}_{\mathrm{f}}, \mathrm{z}_{\mathrm{f}}\right)=(-0.056,0.0,0.170)(\mathrm{m})$ referred to a coordinate system with origin at the center of the reflectarray (see Fig. 6). The corrugations in the feed horn are introduced to minimize cross-pol levels and to reduce sidelobes for lower spillover loss. The corrugated feed-horn is connected to an orthomode transducer (OMT) to operate in the desired single circular polarization. The radiation pattern of the corrugated feed horn has been measured in an anechoic chamber at Universidad Politecnica de Madrid.

Fig. 4(a) shows the corrugated feed-horn mounted in the anechoic chamber. Fig. 4(b) shows the measured copolar and cross-polar radiation patterns for RHCP in the azimuth plane at the extreme and central frequencies of the 19.2-20.2 GHz band. Similar measured results have been obtained for LHCP. In Fig. 4(b) we compare the measured the radiation patterns and simulated radiation patterns at $19.7 \mathrm{GHz}$. The simulations have been carried out by means of CST software. Very good agreement between measurements and simulations is observed for the copolar component of the radiation pattern. However, the crosspolar level of measurements is substantially higher than that of the simulations. These discrepancies are associated to the OMT connected to the feed horn. This OMT introduces a cross-polarization level that is present in the measurement process, but it has not been considered in the simulations. Moreover, since the center frequency of the OMT is close to $20.2 \mathrm{GHz}$, the larger the departure from this frequency, the larger the cross-polarization, which can be 
observed in Fig. 4 (b). It is expected that the large level of cross-polarization introduced by the OMT in the corrugated horn will have a deleterious effect on the cross-polarization of the reflectarray antenna fed by this horn.

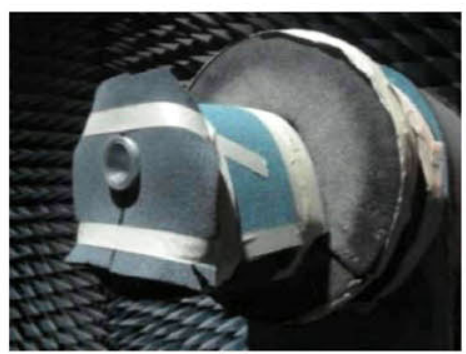

(a)

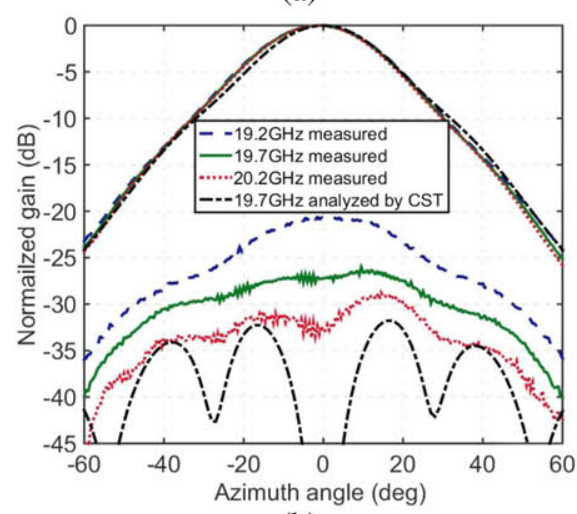

(b)

Fig. 4. (a) Corrugated feed horn in the anechoic chamber. (b) Measured and simulated co- and cross-polar radiation patterns of the corrugated feed horn in the azimuth plane. The results are for RHCP.

Fig. 5 shows the relative illumination level obtained with simulations on the reflectarray aperture at $19.7 \mathrm{GHz}$ when the corrugated feed horn is operating at RHCP. A similar illumination pattern is obtained for LHCP. The results show a level of illumination at the edges of the reflectarray around $7.5 \mathrm{~dB}$ with respect to the maximum level of illumination existing at the center of the reflectarray. This level of illumination provides an acceptable level for spillover loss.

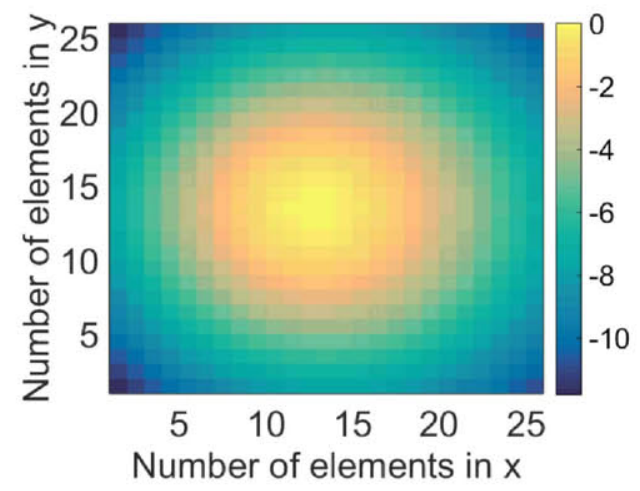

Fig. 5. Simulation results for the relative illumination level obtained on the reflectarray aperture at $19.7 \mathrm{GHz}$ when the aperture is fed by the corrugated horn operating at RHCP.

\section{B. Reflectarray design to generate the same pencil beam for both RHCP and LHCP}

The planar reflectarray consists of $25 \times 25$ elements arranged in a square grid. Since the dimensions of the cells used in Section II are $7.2 \mathrm{~mm} \times 7.2 \mathrm{~mm}$, the total dimensions of the reflectarray are $18 \mathrm{~cm} \times 18 \mathrm{~cm}$. In a preliminary step, we have designed a dual linear polarization reflectarray with elements of the type shown in Fig. 1(a) without rotated dipoles at a center frequency of $19.7 \mathrm{GHz}$ by following the design procedure described in [8]. This reflectarray is intended to produce a focused beam in the direction given by $\phi_{\text {beam }}=0^{\circ}$ and $\theta_{\text {beam }}=19^{\circ}$ for both linear $\mathrm{X}$ and $\mathrm{Y}$-polarization parallel to the axis $\mathrm{X}_{\mathrm{RA}}-\mathrm{Y}_{\mathrm{RA}}$ of Fig. 6 .

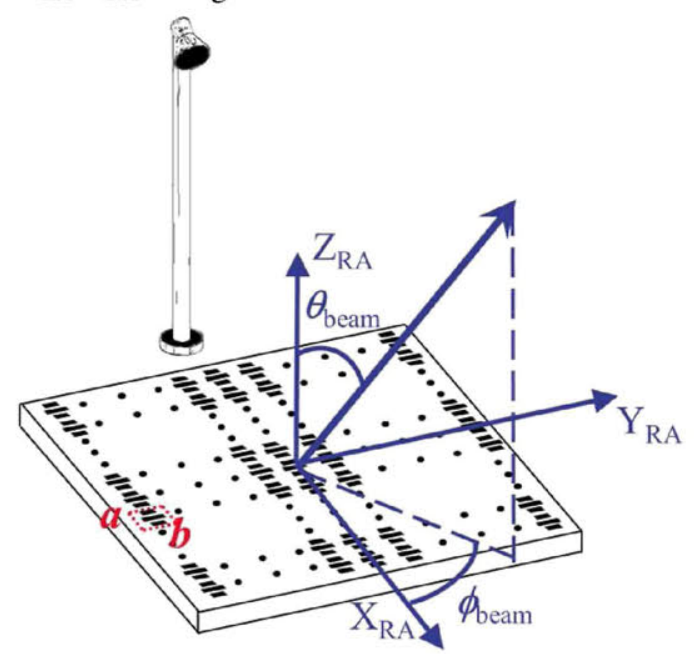

Fig. 6. Reflectarray antenna without rotated dipoles. Coordinate system with origin at the reflectarray center and beam direction.

Fig. 7(a) and (b) show the required phase shift on the elements of the reflectarray to generate a pencil beam in the desired direction at $19.7 \mathrm{GHz}$ for $\mathrm{X}$ and Y-polarization respectively [4]. The phase-shifts enforced in each element for one linear polarization have been deliberately shifted $180^{\circ}$ from those enforced in the orthogonal polarization since this phase difference between the two linear polarizations is a necessary requirement for the phase adjustment in $\mathrm{CP}$ by means of element rotation as shown in Eqns. (3) and (5) of [21].

The reflectarray has been designed at a single frequency 19.7 $\mathrm{GHz}$ by keeping the relative lengths of dipoles defined in section II, and by adjusting the lengths of each set of parallel dipoles to match the phase distribution required in each linear polarization. The lengths of the dipoles are found by a zero finding routine that iteratively calls the MoM-SD analysis tool. In this design process, local periodicity is assumed and the angle of incidence in each element of the reflectarray is explicitly considered.

Even though the lengths of dipoles have been adjusted to generate a pencil beam in the same direction for both $\mathrm{X}$ and Y-polarizations parallel to the periodic cell, the reflectarray will also generate a pencil beam in the same direction for both RHCP and LHCP when it is fed by the circularly polarized corrugated horn described in subsection III.A. 


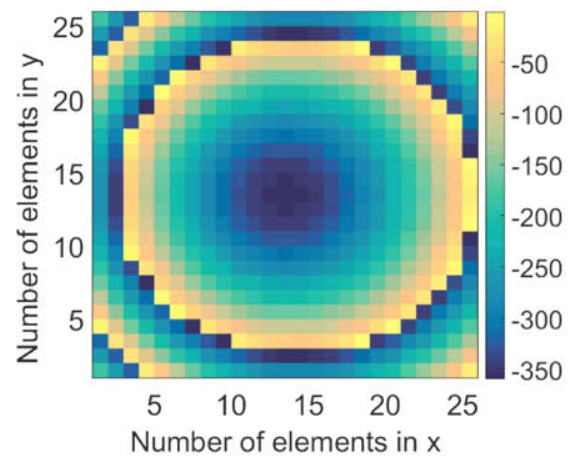

(a)



(b)

Fig. 7. Required phase-shift on the elements of the reflectarray to generate a pencil beam at $19.7 \mathrm{GHz}$ in the direction given by $\phi_{\text {beam }}=0^{\circ}$ and $\theta_{\text {beam }}=19^{\circ}$ (a) for X-polarization and (b) for Y-polarization.

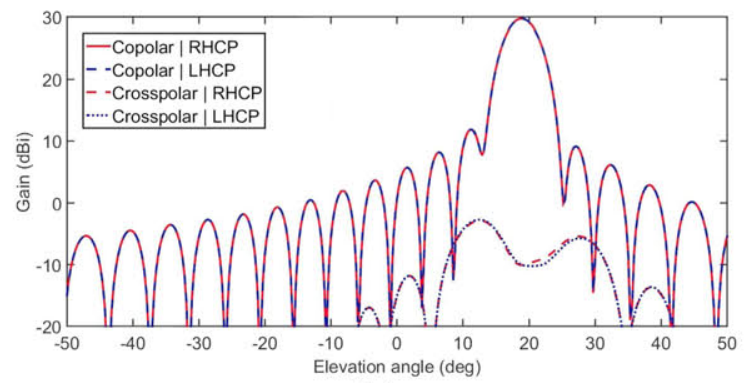

(a)

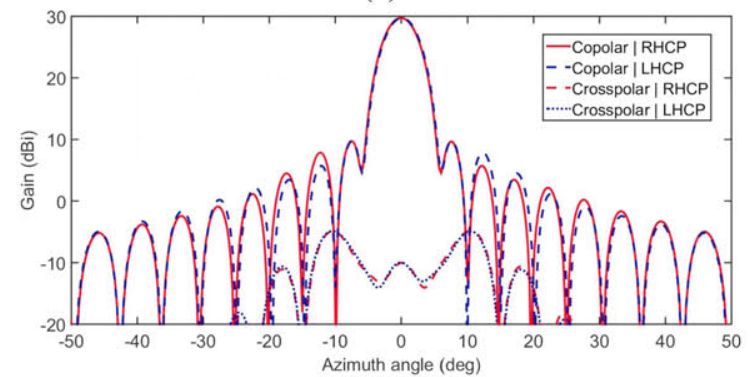

(b)

Fig. 8. Main cuts of the radiation patterns generated at $19.7 \mathrm{GHz}$ by the reflectarray designed for dual linear polarization in the case where the reflectarray is illuminated by the corrugated circularly polarized feed horn. (a) Elevation cut. (b) Azimuth cut.

Fig. 8 shows the elevation and azimuth cuts of the computed radiation patterns in both CPs at $19.7 \mathrm{GHz}$ when the reflectarray is fed by the corrugated horn. These radiation patterns are computed in terms of the electric and magnetic currents existing on the surface of each reflectarray element (technique II in [22]), and these currents are obtained by means of the home-made MoM-SD numerical tool under the local periodicity assumption [8]. The antenna gain is computed in terms of the power radiated by the corrugated horn, and the radiation patterns are computed with reference to the isotropic antenna (in $\mathrm{dBi}$ ) [4]. The numerical results obtained for the radiation patterns indicate a gain of 29.55 dBi (i.e. an efficiency of $66 \%$ ) at $19.7 \mathrm{GHz}$, a $3 \mathrm{~dB}$-beamwidth of $4.6^{\circ}$ and very low cross polarization $(35 \mathrm{~dB}$ and $40 \mathrm{~dB}$ below the maximum of the co-polar beam in the elevation and azimuth plane respectively).

\section{Application of variable rotation technique to separate the pencil beams of RHCP and LHCP}

According to Eqn. (3.1) of [4], the pencil beam radiated by the reflectarray of Subsection III.B will slightly modify its pointing direction from $\theta_{\text {beam }}$ to $\theta_{\text {beam }}+\Delta \theta_{\text {beam }}$ for $\phi_{\text {beam }}=0^{\circ}$ ( $\Delta \theta_{\text {beam }}<<$ is assumed) if the phase of the reflected electric field on the reflectarray aperture is shifted in each element of the reflectarray by the following amount:

$$
\Delta \phi_{i} \approx-k_{0} \sin \left(\Delta \theta_{\text {beam }}\right) \cos \left(\theta_{\text {beam }}\right) x_{i}
$$

where $k_{0}$ is free space propagation constant, and $x_{i}$ is the $\mathrm{x}$ coordinate of the $i$ th-element of the reflectarray in the coordinate system shown in Fig. 6.

Fig. 9(a) and (b) show the phase shifts of $R_{\text {RHCP,RHCP }}$ and $\mathrm{R}_{\text {LHCP,LHCP }}$ that would be required on the elements of the reflectarray designed in Subsection III.B in order to obtain modifications in the main beam directions of $\Delta \theta_{\text {beam }}=2.3^{\circ}$ for LHCP and $\Delta \theta_{\text {beam }}=-2.3^{\circ}$ for RHCP at $19.7 \mathrm{GHz}$ respectively. The required phase shifts are obtained from (6). Since the original design of the reflectarray provides a $3 \mathrm{~dB}$-beamwidth of $4.6^{\circ}$, the angular displacements $\Delta \theta_{\text {beam }}=2.3^{\circ}$ and $\Delta \theta_{\text {beam }}=-$ $2.3^{\circ}$ will produce separate beams that intersect at the point of 3 $\mathrm{dB}$ gain drop.

Eqn. (6) and Fig. 9(a) and (b) clearly indicate that if the values of $\Delta \theta_{\text {beam }}$ for LHCP and RHCP are of equal magnitude and opposite sign, the phase shifts in each reflectarray element for LHCP and RHCP also have to be of the same magnitude and opposite sign, i. e., the phase shifts in the $i$ th-element of the reflectarray have to fulfill the following condition

$$
\Delta \phi_{i, \mathrm{RHCP}}=-\Delta \phi_{i, \mathrm{LHCP}}
$$

If we now have a look at Fig. 3(b), we see that condition (7) is roughly fulfilled when the element of Fig. 1(a) is rotated an angle $\alpha_{\text {rot }}$ as shown in Fig. 1(b). Therefore, we can take advantage of the dipoles rotations in each of the elements of the reflectarray of Subsection III.B to fulfill condition (7).

In order to separate the main beams of LHCP and RHCP in the reflectarray designed in Subsection III.B, the angle of rotation $\alpha_{\text {rot }}$ has been adjusted in each element by means of curves such as those of Fig. 3(b) in order to match the required phase-shifts imposed by Fig. 9(a) and (b). The required 
distribution of angles of rotation is plotted in Fig. 10(a). Note that even though the phase shifts shown in Fig. 9(a) and (b) do not vary within the same column -i. e., among the elements with the same value of $x_{i}$ - in accordance with Eqn. (6), the required values of $\alpha_{\text {rot }}$ plotted in Fig. 10(a) do vary within the same column. And this is because the required values of $\alpha_{\text {rot }}$ for a certain phase shift depend on the angles of incidence and on the dimensions of the dipoles, which vary from one element of the reflectarray to another. Therefore, a figure such as Fig. 3(b) has been generated for each reflectarray element considering its particular angles of incidence and its particular dipoles dimensions. Fig. 10(b) shows the distribution of rotation angles that is obtained when Eqn. (5) is assumed to be valid. Please note that in this latter case the values of $\alpha_{\text {rot }}$ do not vary within the same column since a linear relation is assumed between the phases of $\mathrm{R}_{\mathrm{LHCP}, \mathrm{LHCP}}$ and $\mathrm{R}_{\mathrm{RHCP}, \mathrm{RHCP}}$ and $\alpha_{\text {rot }}$. However, the values of $\alpha_{\text {rot }}$ plotted in Fig. 10(a), which are virtually exact, clearly show that Eqn. (5) is a coarse approximation. One alternative way to fulfill condition (7) while keeping the distribution of rotation angles of Fig. 10(b) is to adjust the dipole lengths after rotation in each element, which is the approach followed in [3].



Number of element in $\mathrm{x}$

(a)

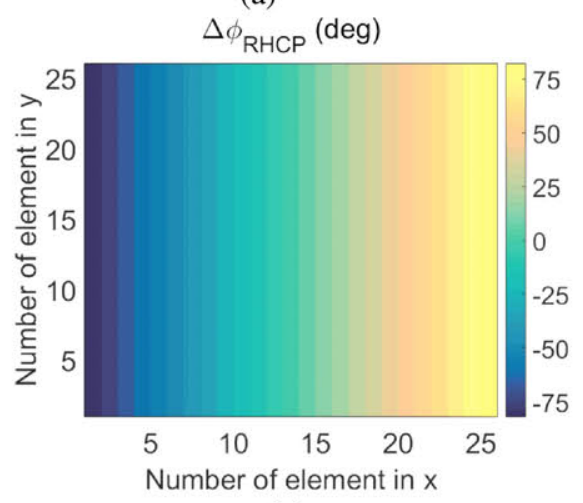

(b)

Fig. 9. Shift in the phases of $R_{\text {LHCP,LHCP }}$ and $R_{\text {RHCP,RHCP }}$ for the reflectarray designed in Subsection III.B that are required for (a) a modification $\Delta \theta_{\text {bean }}=2.3^{\circ}$ in the main beam direction of LHCP at $19.7 \mathrm{GHz}$ and (b) a modification $\Delta \theta_{\text {beam }}=-2.3^{\circ}$ in the main beam direction of RHCP at $19.7 \mathrm{GHz}$.

The reflectarray with separate main beam directions in RHCP and LHCP that has been designed in accordance with the distribution of rotation angles shown in Fig. 10(a) has been manufactured, and their radiation patterns have been measured. The experimental results will be shown in the next section.
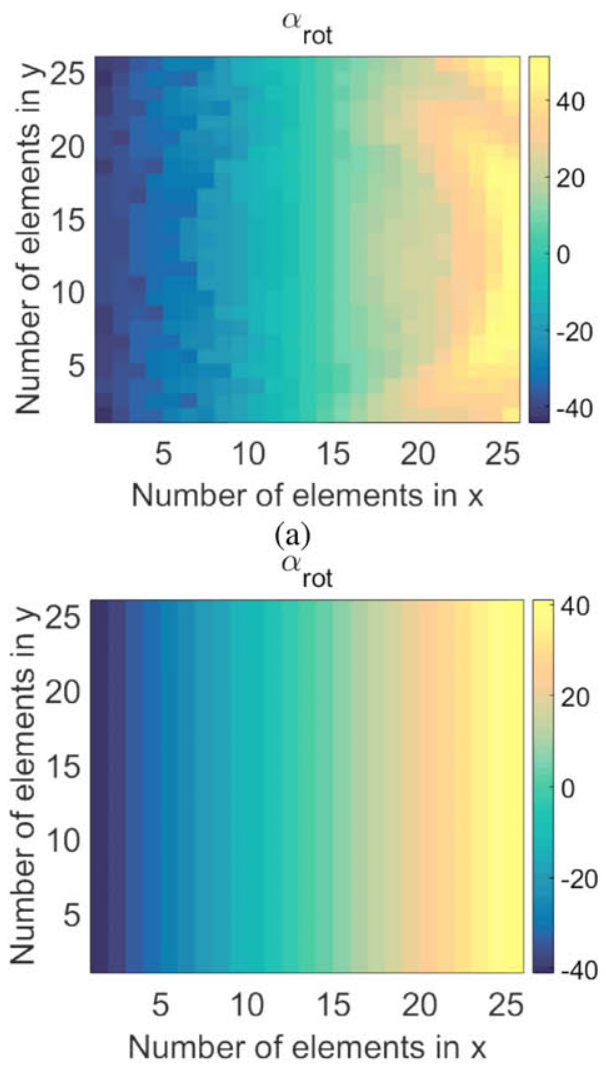

(b)

Fig. 10. Rotation angle $\alpha_{\text {rot }}$ required for each element of the reflectarray designed in Subsection III.B in order to fit the phase shifts shown in Figs. 9(a) and 9(b) for the phases of $R_{\text {RHсP RHCP }}$ and $R_{\text {Ннср.нсP respectively. (a) }}$ Distribution of rotation angles based on Fig. 3(b) for each reflectarray element considering the exact incidence angles. (b) Distribution of rotation angles based on Eqn. (5).

\section{EXPERIMENTAL RESULTS}

The reflectarray with the distribution of rotation angles shown in Fig. 10(a) has been manufactured and its radiation patterns have been measured in a compact range system (see Fig. 11) at Universidad Politecnica de Madrid. The measurements have been carried out at the extreme and center frequencies of the band 19.2-20.2 GHz.

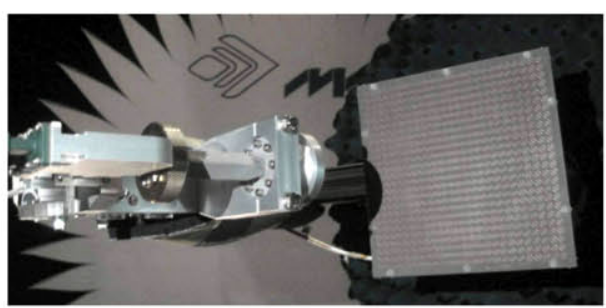

Fig. 11. Reflectarray prototype.

Fig. 12 shows the elevation cut of the simulated and measured radiation patterns provided by the reflectarray prototype at $19.7 \mathrm{GHz}$. 


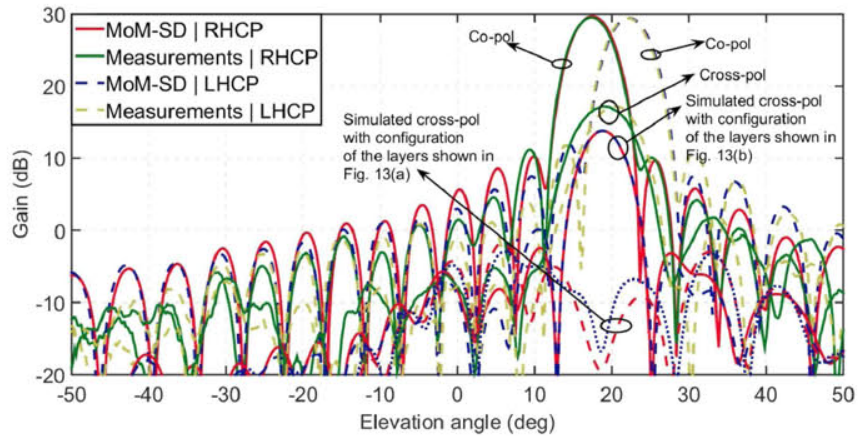

Fig. 12. Measured and simulated elevation cuts for the radiation patterns of the reflectarray designed with separate beams for RHCP and LHCP. The results are presented for a frequency of $19.7 \mathrm{GHz}$. Simulated crosspolarization results are presented for the two different layer configurations of Fig. 13.

Very good agreement between measured and simulated copolar radiation patterns are observed in Fig. 12. In fact, the direction of the main beams for both LHCP and RHCP are shifted $+/-2.3^{\circ}$ with respect to the direction of the main beams shown in Fig. 8(a), which is the final goal of the design procedure described in Subsection III.C. Fig. 12 clearly demonstrates the beam directions of dual CPs can be separated with a planar reflectarray. Furthermore, a second planar reflectarray was designed and measured in which the distribution of rotation angles of Fig. 10(b) was used and the dimensions of the dipoles were subsequently adjusted to fulfill condition (7), which is the approach followed in [3] for the design of a reflectarray with separate $\mathrm{CP}$ beams on a curved surface. In spite of being planar, this second reflectarray also demonstrated the capability of beam separation for LHCP and RHCP in accordance with the design specifications.

Fig. 12 shows the maximum level of cross-polarization is about $15 \mathrm{~dB}$ below the maximum gain in the main beams both in the measured and simulated results, which indicates a large cross-polarization level. This is attributed to an error during the fabrication of the prototype. Whereas the design of the mask (including the choice of the rotation angle and the dipole lengths for each element) was carried out assuming the dipoles were printed at both sides of the upper dielectric layer as shown in Fig. 13(a) (and in Fig. 1), in the manufactured prototype the upper dipoles were printed on the upper layer and the lower dipoles were printed on the separator, which is the configuration shown in Fig. 13(b). This manufacturing error introduces small phase errors that practically do not affect the copolar component of radiation, but it has a significant effect on the cross-polar component as shown in Fig. 12. In fact, this figure indicates the simulated levels of cross-polarization for the correct configuration of Fig. 13(a) are substantially lower than those obtained for the incorrect configuration of Fig. 13(b). These two sets of crosspolarization results were obtained with the dipole dimensions and rotation angles that resulted from the design the antenna under the assumption that the substrate was that of the configuration of Fig. 13(a). The good agreement between the cross-polarization measurements and the cross-polarization simulations obtained with the incorrect configuration of Fig. 13(b) indicates that the high level of cross-polarization of the manufactured breadboard is mainly due to the incorrect position of the dipoles in the fabricated antenna with respect to that assumed in the original design. We would like to add that owing to the cross-polar levels introduced by the OMT which are not included in the design, levels of cross-polarizations around $20 \mathrm{~dB}$ below the maximum gain should be expected in the measurements, even though errors had not been made in the design of the prototype.

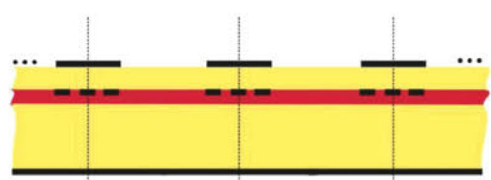

(a)

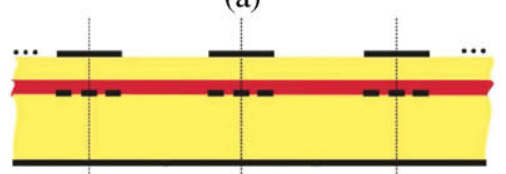

(b)

Fig. 13. Side view of the designed reflectarray. The metallizations are shown in black color, the upper layer and the separator are shown in yellow color, and the thermoplastic bonding film, in red color. (a) Correct configurations of dipoles and layers. (b) Incorrect configuration of dipoles and layers.

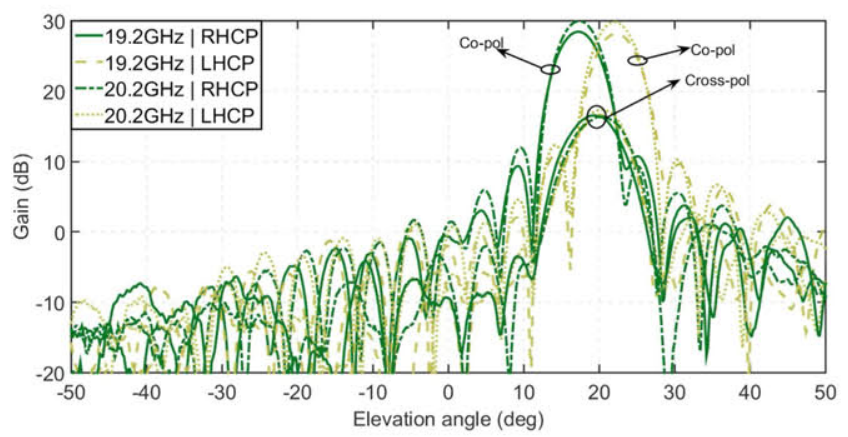

Fig. 14. Elevation cuts of the measured radiation patterns for the manufactured prototype at 19.2 and $20.2 \mathrm{GHz}$.



Fig. 15. Variation of measured gain as a function of frequency.

Fig. 14 shows the measured radiation patterns of the prototype in the elevation plane at the extreme frequencies of the band 19.2-20.2GHz. A gain drop around 1.5dB is observed at $19.2 \mathrm{GHz}$. It has been found that this gain drop can be reduced if the reflectarray is redesigned at a frequency slightly lower than $19.7 \mathrm{GHz}$ (e.g., at a frequency of $19.5 \mathrm{GHz}$ ). The 
high levels of the measured cross-polar radiation patterns in Fig. 14 are again attributed to the errors in the design process described in the previous paragraph. Fig. 15 shows the measured values of gain as a function of the frequency for the RCHP and LHCP main beams. The gain is measured at the fixed angular directions $\theta_{\text {beam }}-\Delta \theta_{\text {beam }}=16.7^{\circ}$ for RHCP and $\theta_{\text {beam }}+\Delta \theta_{\text {beam }}=21.3^{\circ}$ for LHCP. The prototype exhibits $7.6 \%$ bandwidth for $1.5 \mathrm{~dB}$ gain variation, which covers the desired frequency band of operation $19.2-20.2 \mathrm{GHz}$.

The next table summarizes the performance of the manufactured prototype in the whole frequency band.

Table I: Summary of performance of the manufactured prototype

\begin{tabular}{|c|c|c|c|c|c|}
\hline $\begin{array}{c}\theta_{\text {beam }} \text { at } \\
19.7 \mathrm{GHz} \\
(\mathrm{CP})\end{array}$ & $\begin{array}{c}\text { Gain at } \\
19.7 \mathrm{GHz}\end{array}$ & $\begin{array}{c}\text { Bandwidth } \\
1.5 \mathrm{~dB} \text { gain } \\
\text { variation }\end{array}$ & $\begin{array}{c}\text { Antenna } \\
\text { Efficiency } \\
\text { at } 19.7 \mathrm{GHz}\end{array}$ & $\begin{array}{c}\text { Maximum } \\
\text { side lobe } \\
\text { level }\end{array}$ & $\begin{array}{c}\text { Cross-polar } \\
\text { discrimination } \\
\text { at } 19.7 \mathrm{GHz}\end{array}$ \\
\hline $\begin{array}{c}16.7^{\circ} \\
(\mathrm{RHCP})\end{array}$ & $29.39 \mathrm{~dB}$ & $7.6 \%$ & $63 \%$ & $12.36 \mathrm{~dB}$ & $13.27 \mathrm{~dB}$ \\
\hline $\begin{array}{c}21.3^{\circ} \\
(\mathrm{LHCP})\end{array}$ & $29.52 \mathrm{~dB}$ & $7.6 \%$ & $66 \%$ & $11.98 \mathrm{~dB}$ & $13.12 \mathrm{~dB}$ \\
\hline
\end{tabular}

\section{CONCLUSION}

A procedure has been presented to separate the beams of orthogonal $\mathrm{CPs}$ in a reflectarray antenna by means of variable rotations of the reflectarray elements. A planar $18 \mathrm{~cm} \mathrm{x} 18 \mathrm{~cm}$ prototype, capable of generating adjacent pencil beams for orthogonal CPs in Ka-band $(19.2-20.2 \mathrm{GHz})$ has been designed, manufactured and measured as a proof-of-concept to demonstrate the possibility of separating the CP beams. Good agreement between measured and simulated copolar radiation patterns has been observed. The copolar radiation patterns exhibit main beams pointing at $16.7^{\circ}$ for RHCP and $21.3^{\circ}$ for LHCP, with $1.5 \mathrm{~dB}$ gain variation and $66 \%$ of efficiency in the whole frequency band 19.2-20.2GHz. Large cross-polarization levels have been detected in the measured results $(15 \mathrm{~dB}$ below the gain maxima), which are attributed to an error in the location of the bonding layer during the fabrication of the measured prototype.

\section{REFERENCES}

[1] S. K. Rao, "Parametric design and analysis of multiple-beam reflector antennas for satellite communications," IEEE Antennas Propag. Mag., vol. 45, no. 4, pp. 26-34, 2003.

[2] S. K. Rao and M. Q. Tang, "Stepped-reflector antenna for dual-band multiple beam satellite communications payloads," IEEE Trans. Antennas Propag., vol. 54, no. 3, pp. 801-811, 2006.

[3] M. Zhou and S. B. Sørensen, "Multi-spot beam reflectarrays for satellite telecommunications applications in Ka-band," in Proc. 10h Eur. Conf. Antennas \& Propag, Davos, Switzerland, April 2016.

[4] J. Huang and J. A. Encinar, Reflectarray antennas. Piscataway, NJ/New York: IEEE Press/Wiley, 2008.

[5] W. A. Imbriale, S. Gao and L. Boccia, Space Antenna Handbook, John Wiley \& Sons, 2012.

[6] J. A. Encinar et al., "Dual-polarization dual-coverage reflectarray for space applications", IEEE Trans. Antennas Propagat., vol. 54, no. 10, pp. 2827-2837, October 2006.

[7] S. R. Rengarajan, "Reflectarrays of rectangular microstrip patches for dual-polarization dual-beam radar interferometers", Progress In Electromagnetics Research, Vol. 133, 1-15, 2013.

[8] R. Florencio, J. A. Encinar, R. R. Boix, and G. Pérez Palomino, "Dualpolarization reflectarray made of cells with two orthogonal sets of parallel dipoles for bandwidth and cross-polarization improvement," IET Microw. Antennas Propag, Vol. 8, Iss. 15, pp.1389-1397, 2014.
[9] T. Smith, U. V. Gothelf, O. S. Kim, and O. Breinbjerg, "Design, manufacturing, and testing of a $20 / 30 \mathrm{GHz}$ dual-band circularly polarized reflectarray antenna in submission," IEEE Antennas Wireless Propag. Lett., vol. 12, pp. 1480-1483, 2013.

[10] R. Deng, Y. Mao, S. Xu, and F. Yang, "A single-layer dual-band circularly polarized reflectarray with high aperture efficiency," IEEE Trans. Antennas Propagat, vol. 63, no.7, pp. 3317-3320, July 2015.

[11] J. A. Encinar, "Design of a dual frequency reflectarray using stacked patches of variable size," Electronic Letters, vol. 32, no. 12, pp. 10491050 , June 1996

[12] M. R. Chaharmir, J. Shaker, and H. Legay, "Dual-band Ka/X reflectarray with broadband loop elements," in Microwaves, Antennas \& Propag., IET, vol. 4, no. 2, pp. 225-231, Feb. 2010.

[13] T. Smith, U. V. Gothelf, O. S. Kim, and O. Breinbjerg, "An FSS-Backed $20 / 30 \mathrm{GHz}$ circularly polarized reflectarray for a shared aperture L- and Ka-band satellite communication antenna," IEEE Trans. Antennas Propagat., vol. 62, no. 2, pp. 661-668, Feb. 2014.

[14] E. Martinez-de-Rioja, J. A. Encinar, R. Florencio, and R. R Boix, "Reflectarray in $\mathrm{K}$ and $\mathrm{Ka}$ bands with independent beams in each polarization," in Proc. IEEE Int. Symp. Antennas Propag., Fajardo, Puerto Rico, 2016, pp. 1199-1200.

[15] E. Martinez-de-Rioja, J. A. Encinar, M. Barba, R. Florencio, R. R. Boix and V. Losada "Dual polarized reflectarray transmit antenna for operation in $\mathrm{Ku}$ and Ka-bands with independent feeds," IEEE Trans. Antennas Propagat., vol. 65, no. 6, pp. 3241-3246, Jun. 2017.

[16] S. Mener, R. Gillard, R. Sauleau, C. Cheymol and P. Potier, "Unit Cell for Reflectarrays Operating With Independent Dual Circular Polarizations," in IEEE Antennas and Wireless Propagation Letters, vol. 13, pp. 1176-1179, 2014.

[17] S. Mener, R. Gillard, R. Sauleau, A. Bellion and P. Potier, "Dual Circularly Polarized Reflectarray With Independent Control of Polarizations," in IEEE Transactions on Antennas and Propagation, vol. 63, no. 4, pp. 1877-1881, April 2015.

[18] R. Florencio, R. R. Boix, E. Carrasco, J. A. Encinar, and V. Losada, "Efficient numerical tool for the analysis and design of reflectarrays based on cells with three parallel dipoles", Microw. Opt. Technol. Lett., vol. 55 , no. 6, pp. 1212-1216, June 2013.

[19] C. K. Aanandan, P. Debernardi, R. Orta, R. Tascone, and D. Trinchero, "Problem-matched basis functions for moment method analysis- an application to reflection gratings", IEEE Transactions on Antennas and Propagation, vol. 48, no. 1, pp. 35-40, Jan. 2000.

[20] Y. Rahmat-Samii., "Useful coordinate transformation for antenna applications," IEEE Trans. Antennas Propagat., vol. 27, no. 4, pp. 571574, Jul. 1979.

[21] J. Huang, R. J. Pogorzelski, "A Ka-band microstrip reflectarray with elements having variable rotation angles", IEEE Trans. Antennas Propagat., vol. AP-46, pp. 650-656, May 1998.

[22] M. Zhou, S. B. Sorensen, O. S. Kim, E. Jorgensen, P. Meincke, O. S. Kim and O. Breinbjerg, "An accurate technique for calculation of radiation from printed reflectarrays," IEEE Trans. Antennas Propagat., vol. 10, pp. 1081-1084, Oet. 2011. 